Article

\title{
New Simpson type method for solving nonlinear equations
}

\author{
U. K. Qureshi ${ }^{1, *}$, A. A. Shaikhi ${ }^{2}$, F. K. Shaikh ${ }^{2}$, S. K. Hazarewal ${ }^{2}$ and T. A. Laghari ${ }^{2}$ \\ 1 Department of Business Administration, Shaheed Benazir Bhutto University, Sanghar, Sindh, Pakistan. \\ 2 Department of Basic Sciences and Related Studies, Mehran University of Engineering and Technology Jamshoro, \\ Sindh, Pakistan. \\ * Correspondence: umair.khalidsng@sbbusba.edu.pk
}

Academic Editor: Jafar Biazar

Received: 10 December 2020; Accepted: 12 January 2021; Published: 16 March 2021.

\begin{abstract}
Finding root of a nonlinear equation is one of the most important problems in the real world, which arises in the applied sciences and engineering. The researchers developed many numerical methods for estimating roots of nonlinear equations. The this paper, we proposed a new Simpson type method with the help of Simpson 1/3rd rule. It has been proved that the convergence order of the proposed method is two. Some numerical examples are solved to validate the proposed method by using C++/MATLAB and EXCEL. The performance of proposed method is better than the existing ones.
\end{abstract}

Keywords: Nonlinear equation; Simpson 1/3rd rule; Numerical integration; Convergence.

MSC: 58C30; 90C30.

\section{Introduction}

Q uadrature is a historical term which means the process of determining area and is used to computed the value of definite integrals by Newton cotes formula. Indeed, Newton cotes formula is also known as quadrature rule. Newton cotes formula is developed by Isaac Newton and Roger Cotes. Five basic commonly used Quadrature rules for numerical integration are Trapezoidal rule, Simpson 1/3 rule, Simpson 3/8 rule, Boole's rule and Weddle's rule.

Many scientist took interest and developed numerous techniques for numerical integration with the help of mid-point, arithmetic mean, geometric mean, harmonic mean and heroinic mean [1-3]. Now a days, estimating a root of nonlinear equations are one of the most important topics and many numerical techniques have been developed so far [4-6]. The famous iterative methods for finding roots of nonlinear equations are; the Newton's method;

$$
x_{n+1}=x_{n}-\frac{f\left(x_{n}\right)}{f^{\prime}\left(x_{n}\right)}
$$

the Steffensen method;

$$
x_{n+1}=x_{n}-\frac{f^{2}\left(x_{n}\right)}{f\left(x_{n}+f\left(x_{n}\right)\right)+f\left(x_{n}\right)}
$$

the Trapezoidal method;

$$
x_{n+1}=x_{n}-\frac{2 f\left(x_{n}\right)}{f^{\prime}\left(x_{n}\right)+f^{\prime}\left(x_{n}+f\left(x_{n}\right)\right)}
$$

and the Quadrature method;

$$
x_{n+1}=x_{n}-\frac{6 f\left(x_{n}\right)}{f^{\prime}\left(x_{n}\right)+4 f^{\prime}\left(x_{n}+f\left(x_{n}\right)\right)+f^{\prime}\left(x_{n}+2 f\left(x_{n}\right)\right)} .
$$

The aim of this paper is to develop a new numerical method called 'Simpson type method' by using quadrature rule for solving nonlinear equations. The proposed Simpson type method is based on Simpson $1 / 3$ rd rule which converges quadratically. The proposed Simpson type method is compared with Newton Raphson method, Steffensen method, Quadrature method and Trapezoidal method [8-10]. 


\section{Simpson algorithm}

This segment developed a Simpson type method for solving nonlinear equations with the help of Simpson $1 / 3$ rd rule. The Simpson $1 / 3$ rd rule is

$$
\int_{x_{0}}^{x} f(x) d x=\frac{h}{3}\left[f\left(x_{0}\right)+4 f\left(x_{1}\right)+f\left(x_{2}\right)\right] .
$$

Taking derivative, we have

$$
\int_{x_{0}}^{x} f^{\prime}(x) d x=\frac{h}{3}\left[f^{\prime}\left(x_{0}\right)+4 f^{\prime}\left(x_{1}\right)+f^{\prime}\left(x_{2}\right)\right]
$$

The placement of certain integration, we get

$$
f(x)=f\left(x_{0}\right)+\frac{h}{3}\left[f^{\prime}\left(x_{0}\right)+4 f^{\prime}\left(x_{1}\right)+f^{\prime}\left(x_{2}\right)\right],
$$

where $n=2$ and $h=\frac{x-x_{0}}{2}$.

Substitute the value of $h$ in (1), we have

$$
f(x)=f\left(x_{0}\right)+\left(x-x_{0}\right) \frac{f^{\prime}\left(x_{0}\right)+4 f^{\prime}\left(x_{1}\right)+f^{\prime}\left(x_{2}\right)}{6},
$$

where $x_{0}$ is an initial guess, which is near to root $x$. Now using $f(x)=0$, (2) becomes

$$
x=x_{0}-\frac{6 f\left(x_{0}\right)}{f^{\prime}\left(x_{0}\right)+4 f^{\prime}\left(x_{1}\right)+f^{\prime}\left(x_{2}\right)} .
$$

The basic numerical technique [7] is

$$
x_{1}=x_{0}+f\left(x_{0}\right)
$$

or

$$
x_{2}=x_{0}+2 f\left(x_{0}\right)
$$

Substitute (4) and (5) in (3), we get

$$
x=x_{0}-\frac{6 f\left(x_{0}\right)}{f^{\prime}\left(x_{0}\right)+4 f^{\prime}\left(x_{0}+f\left(x_{0}\right)\right)+f^{\prime}\left(x_{0}+2 f\left(x_{0}\right)\right)} .
$$

In general

$$
x_{n+1}=x_{n}-\frac{6 f\left(x_{n}\right)}{f^{\prime}\left(x_{n}\right)+4 f^{\prime}\left(x_{n}+f\left(x_{n}\right)\right)+f^{\prime}\left(x_{n}+2 f\left(x_{n}\right)\right)} .
$$

Equation (6) is the Simpson type method for solving nonlinear equations.

\section{Convergence analysis}

In the following theorem, we prove that the proposed Simpson type method converges quadratically.

Theorem 1. Let $f: D \subseteq R \rightarrow R$ be a sufficiently differentiable function and $a \in D$ be a root of it in the open interval $D$. If $x_{0}$ is sufficiently close to $a$, then the proposed Simpson type method has second-order convergence and satisfies the following error equation:

$$
e_{n+1}=e_{n}^{2}\left[-f^{\prime \prime}(a)+c\left(1+7 f^{\prime}(a)\right]\right.
$$

Proof. Let $a$ be the root of the function $f$. By using Taylor series we have, we have

$$
f\left(x_{n}\right)=f^{\prime}(a)\left(e_{n}+c e_{n}^{2}\right)
$$

implies

$$
f^{\prime}\left(x_{n}\right)=f^{\prime}(a)\left(1+2 c e_{n}+3 c^{2} e_{n}^{2}\right) .
$$

Now

$$
f\left(x_{n}+f\left(x_{n}\right)\right)=f^{\prime}(a)\left[\left(e_{n}+f\left(x_{n}\right)\right)+\left(e_{n}+f\left(x_{n}\right)\right)^{2} c\right] .
$$


By taking derivative of (9), we get

$$
f^{\prime}\left(x_{n}+f\left(x_{n}\right)\right)=f^{\prime}(a)\left[\left(1+f^{\prime}\left(x_{n}\right)\right)+2\left(e_{n}+f\left(x_{n}\right)\right)\left(1+f^{\prime}\left(x_{n}\right)\right) c\right] .
$$

Using (7) and (8) in (10), we get

$$
f^{\prime}\left(x_{n}+f\left(x_{n}\right)\right)=f^{\prime}(a)\left[1+f^{\prime}(a)+2 c e_{n}+6 c e_{n} f^{\prime}(a)+2 c e_{n} f^{\prime 2}(a)\right] .
$$

Now,

$$
f\left(x_{n}+2 f\left(x_{n}\right)\right)=f^{\prime}(a)\left[\left(e_{n}+2 f\left(x_{n}\right)\right)+\left(e_{n}+2 f\left(x_{n}\right)\right)^{2} c\right] .
$$

Taking derivative of (12), we get

$$
f^{\prime}\left(x_{n}+2 f\left(x_{n}\right)\right)=f^{\prime}(a)\left[\left(1+2 f^{\prime}\left(x_{n}\right)\right)+2\left(e_{n}+2 f\left(x_{n}\right)\right)\left(1+2 f^{\prime}\left(x_{n}\right)\right) c\right] .
$$

Using (7) and (8) in (13), we get

$$
f^{\prime}\left(x_{n}+2 f\left(x_{n}\right)\right)=f^{\prime}(a)\left[1+2 f^{\prime}(a)+2 c e_{n}+12 c e_{n} f^{\prime}(a)+8 c e_{n} f^{\prime 2}(a)\right] .
$$

By solving (8), (9) and (14), we get

$$
f^{\prime}\left(x_{n}\right)+f^{\prime}\left(x_{n}+f\left(x_{n}\right)\right)+f^{\prime}\left(x_{n}+2 f\left(x_{n}\right)\right)=6 f^{\prime}(a)\left[1+f^{\prime}(a)+2 c e_{n}+6 c e_{n} f^{\prime}(a)\right] .
$$

Now using (7)-(15) in (6), we get

$$
\begin{aligned}
e_{n+1} & =e_{n}-\frac{6 e_{n} f^{\prime}(a)\left(1+c e_{n}\right)}{6 f^{\prime}(a)\left[1+2 c e_{n}+f^{\prime}(a)+6 c e_{n} f^{\prime}(a)\right]} \\
& =e_{n}-\frac{e_{n}\left(1+c e_{n}\right)}{1+2 c e_{n}+f^{\prime}(a)+6 c e_{n} f^{\prime}(a)} \\
& =e_{n}-e_{n}\left(1+c e_{n}\right)\left[1+2 c e_{n}+f^{\prime}(a)+6 c e_{n} f^{\prime}(a)\right]^{-1} \\
& =e_{n}-e_{n}\left(1+c e_{n}\right)\left[1-2 c e_{n}-f^{\prime}(a)-6 c e_{n} f^{\prime}(a)\right] \\
& =e_{n}-e_{n}\left[1-f^{\prime}(a)-c e_{n}-7 c e_{n} f^{\prime}(a)\right] \\
& =e_{n} f^{\prime}(a)+c e_{n}^{2}\left(1+7 f^{\prime}(a)\right) .
\end{aligned}
$$

Finally, we get

$$
e_{n+1}=-e_{n}^{2} f^{\prime \prime}(a)+c e_{n}^{2}\left(1+7 f^{\prime}(a)\right)
$$

or

$$
e_{n+1}=e_{n}^{2}\left[-f^{\prime \prime}(a)+c\left(1+7 f^{\prime}(a)\right)\right],
$$

where $c=\frac{f^{\prime}(a)}{2 f^{\prime}(a)}$. Hence, the proposed Simpson type method converges quadratically.

\section{Numerical results}

Now, we solve some examples numerically to compare the performance of our proposed Simpson type method by using $\mathrm{C}++$ and EXCEL. We compared our Simpson type method (P S Method) with Newton Raphson method (NR Method), Steffensen method (S Method), Trapezoidal method (T Method), and Quadrature method (Q Method).

Example 1. Consider the nonlinear equation;

$$
\sin ^{2} x-x^{2}+1=0
$$

which represents the mass of the jumper. By taking $x_{0}=1$ as initially guess, the comparison of NR Method, $\mathrm{S}$ Method, T Method, Q Method and P S Method is given in Table 1 and error analysis if given in Table 2. It can be observed from Table 1 that P S Method converges faster to the root of the Equation (18) than NR Method, S Method, T Method and Q Method. The graphical comparison of error analysis is shown in Figure 1. 
Table 1. Numerical results for the Equation (18)

\begin{tabular}{c|c|c|c|c|c} 
Iteration & NR Method & S Method & T Method & Q Method & PS Method \\
\hline 1 & 1.64919 & 1.30491 & 1.24171 & 1.48888 & 1.47631 \\
\hline 2 & 1.43904 & 1.39468 & 1.38049 & 1.40474 & 1.40434 \\
\hline 3 & 1.40539 & 1.40438 & 1.40403 & 1.40449 & 1.40449 \\
\hline 4 & 1.40449 & 1.40449 & 1.40449 & 1.40449 & 1.40449 \\
\hline 5 & 1.40449 & 1.40449 & 1.40449 & 1.40449 & 1.40449 \\
\hline 6 & 1.40449 & 1.40449 & 1.40449 & & \\
\hline 7 & 1.40449 & 1.40449 & 1.40449 & &
\end{tabular}

Table 2. Error analysis of NR Method, S Method, T Method, Q Method and P S Method for the Equation (18)

\begin{tabular}{l|l|l|l|l|l} 
Iteration & NR Method & S Method & T Method & Q Method & P S Method \\
\hline 1 & 0.64919 & 0.30491 & 0.241713 & 0.488877 & 0.476306 \\
\hline 2 & 0.210148 & 0.0897719 & 0.13878 & 0.0841343 & 0.0719708 \\
\hline 3 & 0.0336573 & 0.00969983 & 0.023536 & 0.00025146 & 0.000156053 \\
\hline 4 & 0.000892813 & 0.000109995 & 0.000463009 & $6.74309 \mathrm{e}-009$ & $2.60288 \mathrm{e}-009$ \\
\hline 5 & $6.24721 \mathrm{e}-007$ & $1.40554 \mathrm{e}-008$ & $1.68211 \mathrm{e}-007$ & $2.22045 \mathrm{e}-016$ & $2.22045 \mathrm{e}-016$ \\
\hline 6 & $3.05977 \mathrm{e}-013$ & $4.44089 \mathrm{e}-016$ & $2.24265 \mathrm{e}-014$ & & \\
\hline 7 & $2.22045 \mathrm{e}-016$ & $2.22045 \mathrm{e}-016$ & $2.22045 \mathrm{e}-016$ & &
\end{tabular}

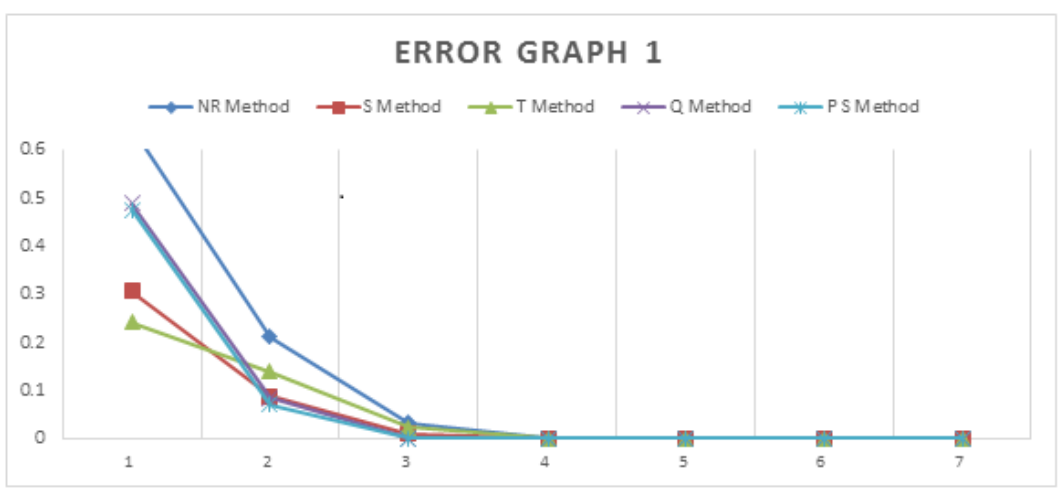

Figure 1. Graphical comparison of error analysis for the Equation (18)

Example 2. Consider the nonlinear equation;

$$
2 x^{2}-5 x-2=0
$$

which represents the diameter of the pipe. By taking $x_{0}=1$ as initially guess, the comparison of NR Method, S Method, T Method, Q Method and P S Method is given in Table 3 and error analysis if given in Table 4. It can be observed from Table 3 that P S Method converges faster to the root of the Equation (19) than NR Method, S Method, T Method and Q Method. The graphical comparison of error analysis is shown in Figure 2.

Table 3. Numerical results for the Equation (19)

\begin{tabular}{c|c|c|c|c|c} 
Iteration & NR Method & S Method & T Method & Q Method & PS Method \\
\hline 1 & 4 & 0.545455 & 0.761905 & 0.166667 & 0.166667 \\
\hline 2 & 1.61905 & 0.172598 & 0.356846 & 0.223958 & 0.223958 \\
\hline 3 & 0.631101 & 0.110105 & 0.112234 & 0.340784 & 0.340784 \\
\hline 4 & 0.371668 & 0.282008 & 0.330164 & 0.350713 & 0.350713 \\
\hline 5 & 0.350916 & 0.343655 & 0.350647 & 0.350781 & 0.350781 \\
\hline 6 & 0.350781 & 0.350696 & 0.350781 & 0.350781 & 0.350781 \\
\hline 7 & 0.350781 & 0.350781 & 0.350781 & & \\
\hline 8 & 0.350781 & 0.350781 & 0.350781 & &
\end{tabular}


Table 4. Error analysis of NR Method, S Method, T Method, Q Method and P S Method for the Equation (19)

\begin{tabular}{l|l|l|l|l|l} 
Iteration & NR Method & S Method & T Method & Q Method & P S Method \\
\hline 1 & 5 & 0.454545 & 0.238095 & 0.833333 & 0.833333 \\
\hline 2 & 2.38095 & 0.372856 & 0.405058 & 0.390625 & 0.390625 \\
\hline 3 & 0.987947 & 0.282704 & 0.469081 & 0.116825 & 0.116825 \\
\hline 4 & 0.259433 & 0.171903 & 0.217929 & 0.00992928 & 0.00992928 \\
\hline 5 & 0.0207519 & 0.0616468 & 0.0204829 & $6.81612 \mathrm{e}-005$ & $6.81612 \mathrm{e}-005$ \\
\hline 6 & 0.000134499 & 0.00704128 & 0.000134488 & $3.19492 \mathrm{e}-009$ & $3.19492 \mathrm{e}-009$ \\
\hline 7 & $5.65037 \mathrm{e}-009$ & $8.46319 \mathrm{e}-005$ & $5.65037 \mathrm{e}-009$ & & \\
\hline 8 & $5.55112 \mathrm{e}-017$ & $1.20896 \mathrm{e}-008$ & $5.55112 \mathrm{e}-017$ & & \\
\hline 9 & & $2.77556 \mathrm{e}-016$ & & &
\end{tabular}

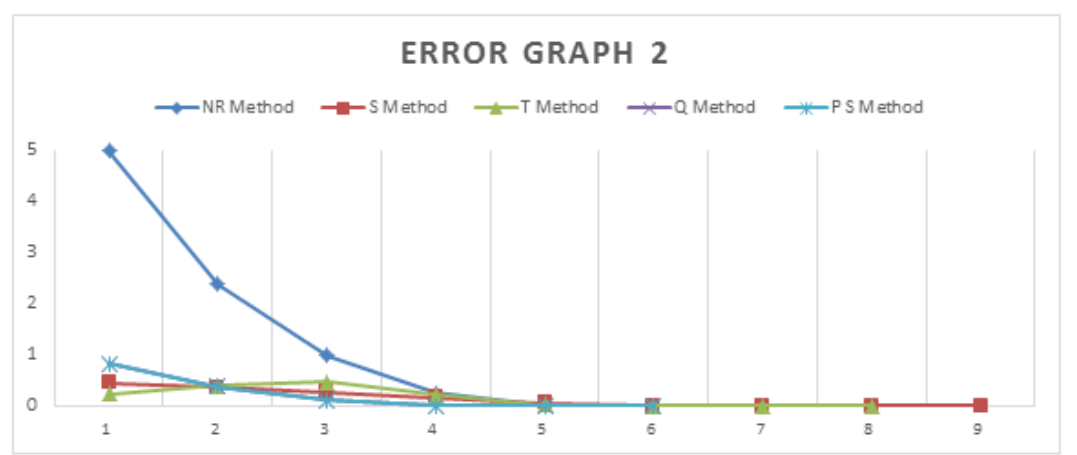

Figure 2. Graphical comparison of error analysis for the Equation (19)

Example 3. Consider the nonlinear equation;

$$
x^{2}-e^{x}=0,
$$

which represents the volume of the gas depends. By taking $x_{0}=1$ as initially guess, the comparison of NR Method, S Method, T Method, Q Method and P S Method is given in Table 5 and error analysis if given in Table 6. It can be observed from Table 5 that P S Method converges faster to the root of the Equation (20) than NR Method, S Method, T Method and Q Method. The graphical comparison of error analysis is shown in Figure 3.

Table 5. Numerical results for the Equation (20)

\begin{tabular}{c|c|c|c|c|c} 
Iteration & NR Method & S Method & T Method & Q Method & PS Method \\
\hline 1 & 1 & 0.6127 & 0.5 & 0.681304 & 0.697119 \\
\hline 2 & 0.733044 & 0.700737 & 0.686102 & 0.703412 & 0.703463 \\
\hline 3 & 0.703808 & 0.703465 & 0.703347 & 0.703467 & 0.703467 \\
\hline 4 & 0.703467 & 0.703467 & 0.703347 & 0.703467 & 0.703467 \\
\hline 5 & 0.703467 & 0.703467 & 0.703347 & & \\
\hline 6 & 0.703467 & & & &
\end{tabular}

Table 6. Error analysis of NR Method, S Method, T Method, Q Method and P S Method for the Equation (20)

\begin{tabular}{l|l|l|l|l|l} 
Iteration & NR Method & S Method & T Method & Q Method & P S Method \\
\hline 1 & 1 & 0.6127 & 0.5 & 0.681304 & 0.697119 \\
\hline 2 & 0.266956 & 0.0880376 & 0.186102 & 0.0221084 & 0.00634409 \\
\hline 3 & 0.0292358 & 0.00272734 & 0.017245 & $5.502 \mathrm{e}-005$ & $4.42231 \mathrm{e}-006$ \\
\hline 4 & 0.000340318 & $2.65374 \mathrm{e}-006$ & 0.000120043 & $3.31265 \mathrm{e}-010$ & $2.13995 \mathrm{e}-012$ \\
\hline 5 & $4.58334 \mathrm{e}-008$ & $2.5131 \mathrm{e}-012$ & $5.70312 \mathrm{e}-009$ & & \\
\hline 7 & $7.77156 \mathrm{e}-016$ & & & &
\end{tabular}




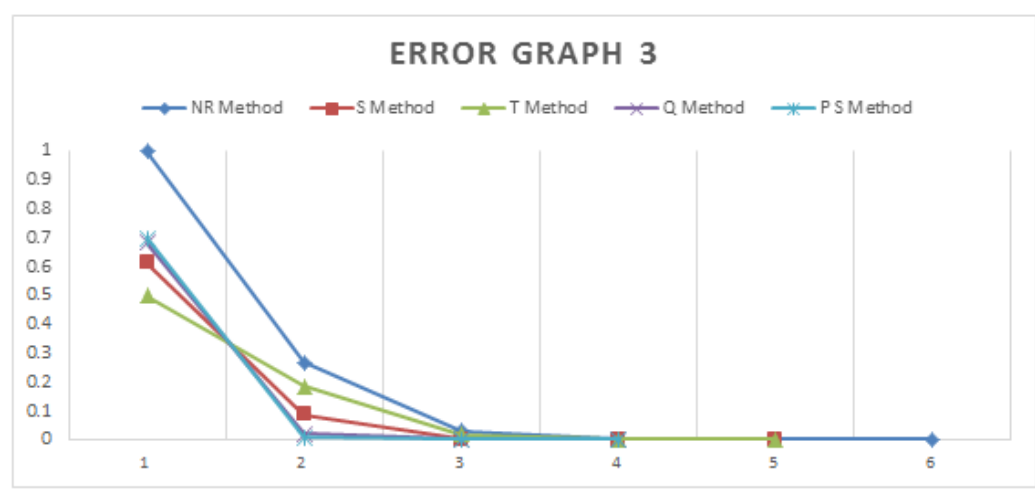

Figure 3. Graphical comparison of error analysis for the Equation (20)

Example 4. Consider the nonlinear equation;

$$
\sin x-x-1=0
$$

which represents the anti-symmetric buckling of a beam. By taking $x_{0}=1$ as initially guess, the comparison of NR Method, S Method, T Method, Q Method and P S Method is given in Table 5 and error analysis if given in Table 8. It can be observed from Table 7 that P S Method converges faster to the root of the Equation (21) than NR Method, S Method, T Method and Q Method. The graphical comparison of error analysis is shown in Figure 4.

Table 7. Numerical results for the Equation (21)

\begin{tabular}{c|c|c|c|c|c} 
Iteration & NR Method & S Method & T Method & Q Method & PS Method \\
\hline 1 & 1.06086 & 2.02963 & 0.751682 & 1.19666 & 2.29644 \\
\hline 2 & 1.26018 & 1.93335 & 1.18987 & 1.75841 & 1.82684 \\
\hline 3 & 2.25674 & 1.93456 & 2.21057 & 1.919 & 1.92833 \\
\hline 4 & 1.96109 & 1.93456 & 4.00889 & 1.93442 & 1.93454 \\
\hline 5 & 1.9348 & 1.93456 & 2.89742 & 1.93456 & 1.93456 \\
\hline 6 & 1.93456 & & 1.96053 & 1.93456 & 1.93456 \\
\hline 7 & 1.93456 & & 1.93434 & 1.93456 & \\
\hline 8 & 1.93456 & & 1.93456 & & \\
\hline 9 & & & 1.93456 & & \\
\hline 10 & & & 1.93456 & &
\end{tabular}

Table 8. Error analysis of NR Method, S Method, T Method, Q Method and P S Method for the Equation (21)

\begin{tabular}{l|l|l|l|l|l} 
Iteration & NR Method & S Method & T Method & Q Method & P S Method \\
\hline 1 & 1.93914 & 5.02963 & 2.24832 & 4.19666 & 5.29644 \\
\hline 2 & 2.32104 & 0.0962742 & 0.43819 & 0.561751 & 0.469605 \\
\hline 3 & 0.996557 & 0.0012114 & 1.0207 & 0.160589 & 0.101488 \\
\hline 4 & 0.29565 & $1.79787 \mathrm{e}-007$ & 6.21945 & 0.0154234 & 0.00621495 \\
\hline 5 & 0.0262884 & $3.9968 \mathrm{e}-015$ & 1.11146 & 0.000140799 & $2.28272 \mathrm{e}-005$ \\
\hline 6 & 0.000236493 & & 0.936891 & $1.1695 \mathrm{e}-008$ & $3.07394 \mathrm{e}-010$ \\
\hline 7 & $1.92751 \mathrm{e}-008$ & & 0.0261916 & $2.22045 \mathrm{e}-016$ & \\
\hline 8 & $2.22045 \mathrm{e}-016$ & & 0.000225115 & & \\
\hline 9 & & & $1.74734 \mathrm{e}-008$ & & \\
\hline 10 & & & $2.22045 \mathrm{e}-016$ & & \\
\hline
\end{tabular}

\section{Conclusion}

We proposed a new numerical method for solving nonlinear equations which is derived from Simpson $1 / 3$ rd rule. It has been proved that the proposed method converges quadratically. We solved different nonlinear equations numerically by using Newton Raphson method, Steffensen method, quadrature method, 


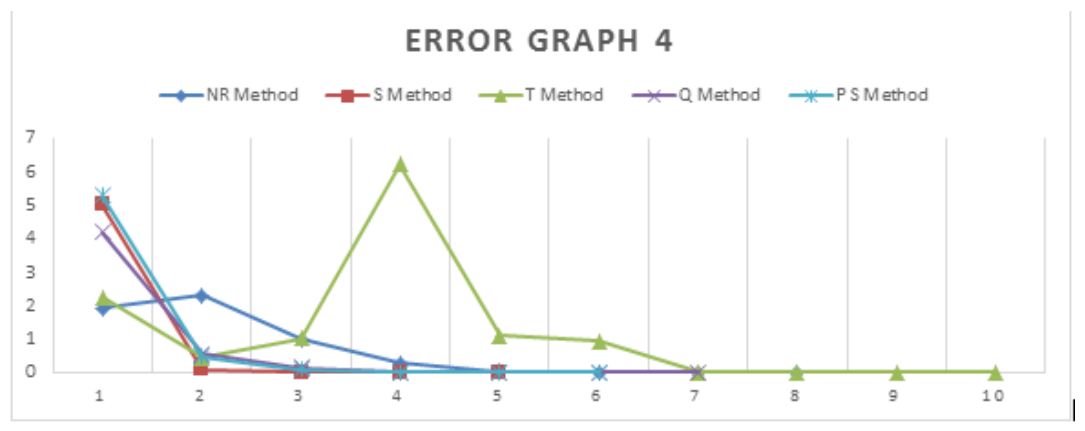

Figure 4. Graphical comparison of error analysis for the Equation (21)

trapezoidal method and our proposed method. The numerical results show that the proposed method is more accurate and faster than Newton Raphson method, Steffensen method, Quadrature method and Trapezoidal method.

Acknowledgments: The authors would like to thank Prof. Dr. Asif Ali Shaikh from the Department of Basic Science and Related Studies, Mehran University of Engineering and Technology, Jamshoro, Sindh, Pakistan for his support.

Conflicts of Interest: "The authors declare no conflict of interest".

\section{References}

[1] Shaikh, M. M., Chandio, M. S., \& Soomro, A. S. (2016). A modified four-point closed mid-point derivative based quadrature rule for numerical integration. Sindh University Research Journal-SURJ (Science Series), 48(2), $389-392$.

[2] Khatri, A., Shaikh, A. A. \& Abro, K. A. (2019). Closed Newton cotes quadrature rules with derivatives. Mathematical Theory and Modeling, 9(5), 65-72.

[3] Perhiyar, M. A., Shah, S. f \& Shaikh, A. A. (2019). Modified Trapezoidal rule based different averages for numerical integration. Mathematical Theory and Modeling, 9(9), 72-75.

[4] Mallah, A. A., Shaikh, A. A. \& Qureshi, S. (2020). An improved root location method for fast convergence of non-linear equations. Mathematical Theory and Modeling, 10(5), 51-57.

[5] Eskandari, H. (2017). Simpson,s method for solution of nonlinear equation. Applied Mathematics, 8(7), 929-933.

[6] Qureshi, U. K. (2018). A new accelerated third-order two-step iterative method for solving nonlinear equations. Mathematical Theory and Modeling, 8(5), 64-68.

[7] Qureshi, U. K., \& Kalhoro, Z. A. (2018). Trapezoidal second order iterated method for solving nonlinear problems. University of Sindh Journal of Information and Communication Technology, 2(2), 111-114.

[8] Qureshi, U. K., Bozdar, I. A., Pirzada, A., \& Arain, M. B. (2019). Quadrature rule based iterative method for the solution of non-linear equations. Proceedings of the Pakistan Academy of Sciences, 56(1), 39-43.

[9] Akram, S., \& Ann, Q. U. (2015). Newton raphson method. International Journal of Scientific E Engineering Research, 6(7), $1748-1752$.

[10] Liu, Z., \& Zhang, H. (2014). Steffensen-type method of super third-order convergence for solving nonlinear equations. Journal of Applied Mathematics and Physics, 2(7), 581-586. 